\title{
Biomarkers for the Early Stages of Clinical Development in Alzheimer's Disease
}

\author{
Régis Bordet ${ }^{1}$, Jean-François Dartigues ${ }^{2}$, Bruno Dubois ${ }^{3}$, Jean-Marie Goehrs ${ }^{4}$, Laura Vernoux ${ }^{5}$, Franck Semah ${ }^{6}$, \\ Florence Pasquier ${ }^{7}$, Claude Bidaut-Mazel ${ }^{8}$ and participants of Round Table $n^{\circ} 3$ of Giens XXV \\ 1 EA 1046, Faculté de Médecine, Université de Lille II, Département Universitaire de Pharmacologie, Lille, France \\ 2 ISPED, Inserm U897, Université Victor Segalen Bordeaux 2, Bordeaux, France \\ 3 Fédération des Maladies du Système Nerveux, Hôpital de la Pitié-Salpétrière, Paris, France \\ 4 Faculté de Médecine Pierre et Marie Curie, Paris, France \\ 5 Innogenetics, Les Ulis, France \\ 6 Service de Médecine Nucléaire et Imagerie Fonctionnelle, CHRU Lille, France \\ 7 Pôle de Neurologie, Hôpital Salengro, CHRU Lille, France \\ 8 GlaxoSmithKline, Marly le Roi, France
}

Text received 4 march 2010; accepted 25 may 2010

\section{Keywords:}

Alzheimer's disease; clinical development; biomarker; imaging

\begin{abstract}
As the failure of several recent Phase III drug development programmes bears witness, the clinical development of "disease-modifying" drugs in Alzheimer's disease has been confronted with challenging methodological difficulties. Taking into account the financial stakes involved taking drug candidates to the Phase III stage of development, and the risk of investing time and resources fruitlessly in the evaluation of poor candidate drugs, the crucial decision remains whether to proceed from Phase II to Phase III (Go/Nogo). The aim of Phase II studies is to select a molecule likely to be effective in Phase III, but also to eliminate candidate-drugs with an inadequate effect. No consensus currently exists on the best possible design of Phase II studies to inform the Go/Nogo decision optimally. The challenges in choosing the best study design relate to the target population, the end-point criteria used, in particular the use of biomarkers, the experimental protocol, and the study duration. The objective of the Round Table (RT) was to gather the opinions of French experts from the academic, industrial, and regulatory world in order to arrive at a consensus recommendation for the best possible design to be used in Phase II studies in Alzheimer's disease.
\end{abstract}

\section{Alzheimer's disease: prodromal phase and dementia}

Consistent with the recommendations of the previous year's RT, the members of the RT emphasised the need to distinguish between Alzheimer's disease as a whole and dementia, which constitutes only one evolutionary stage of the disease. The RT participants suggested that drugs currently undergoing evaluation should target a prodromal or pre-demential phase, although they observed that study protocols declared on www.clinical-trial.gov still have dementia as an inclusion criterion for Alzheimer's disease, even when this is only mild to moderate. The discussion then turned to the idea of selecting an "enriched" population for

\footnotetext{
^ For a list of partiticpants, see end of article.
}

clinical trials, which could lead regulatory authorities to restrict the indication and reimbursement to only this target population. However, participants considered that it was preferable to have a drug that was effective in a restricted population than no effective registered drug at all.

\section{Disease-modifying drugs: evolutionary course and physiopathological effect}

Participants discussed the idea of disease-modifying drugs and considered that such candidate-drugs should be able (i) to modify the evolutionary course of the disease in the longterm; and (ii) to have an effect on the physiopathology of the disease. The difference between a symptomatic effect and a 
disease-modifying effect is that, theoretically, the long-term disease course is altered by the disease-modifying drug, even when it has been discontinued, whereas when treatment that is purely symptomatic is stopped, any clinical benefit ceases. However, the discussion remained open on two points. The first concerned the nature of the physiopathological effect, with questions on whether only activity against specific mechanisms of the disease such as the amyloid cascade or neurofibrillar degeneration should be considered, or whether less specific actions (for example, inflammation, oxidative stress or vascular and metabolic functions) should also be taken into account in the disease-modifying effect. The second point concerned the strategy to adopt in order to demonstrate an effect on the physiopathology of disease. Either this could be done concomitantly with the demonstration of an effect on the disease course, for example by the demonstration of an effect on a relevant physiopathological biomarker, or it could be done subsequently, using a biomarker that is non-specific for progression. Although the European Medicine Agency (EMA) ${ }^{[1]}$ guidelines favour the first solution, it seems reasonable to take into account the stage of development of the drug and to consider the demonstration of a physiopathological mechanism as secondary to the demonstration of a therapeutic effect and not as a prerequisite, particularly because the real mechanism of action of a drug is sometimes different to that imagined when clinical development was initiated. Furthermore, the value of a biomarker as a decisionmaking tool needs to be re-evaluated periodically in light of the data available, as has been evidently the case in the past for other therapeutic areas, such as bone mineral density for osteoporosis or ventricular extrasystoles for prevention of myocardial infarction. ${ }^{[2]}$

\section{Which biomarkers for Alzheimer's disease?}

Because of the difficulties in demonstrating the efficacy of a candidate-drug using only clinical and cognitive tools, biomarkers have become more important over the past few years. A biomarker is defined as a parameter that can be measured in an objective way and that serves to indicate a normal biological process, a pathological process or a pharmacological response to a therapeutic intervention. It can be used as an intermediary assessment criterion ("surrogate endpoint"), when it can be substituted for a clinical assessment criterion. In addition to cognitive and psychobehavioural scales, which are themselves biomarkers of clinical state, genetic, biological, neurophysiological or imaging biomarkers have been proposed during the course of Alzheimer's disease. Some are linked to the physiopathological cascade in Alzheimer's disease and are indicators of specific (amyloid cascade or abnormal phosphorylation of tau protein) or non-specific mechanisms.
Others are markers of cerebral distress and are independent of the underlying physiopathological mechanisms.

Genetic biomarkers have been identified as a result of epidemiological genetic studies in familial and sporadic Alzheimer's disease. The best characterised marker is the allele epsilon4 of the apolipoprotein E gene, which represents both a susceptibility marker for the development of Alzheimer's disease and a stratification tool in terms of therapeutic response. Numerous recently identified genes associated with an increased risk of Alzheimer's disease may be potential markers to integrate into development programmes for new drugs in Alzheimer's disease, since they are potentially associated with different pharmacological responses. However, although they may be useful to define target populations better, they may be less so for the evaluation of therapeutic efficacy.

Progress concerning biological markers has been considerable over the past five years following better characterisation of physiopathological pathways and findings from bioclinical studies. For the main part, these biological markers correspond to components of the specific pathophysiological cascade in Alzheimer's disease, which can be measured in the cerebrospinal fluid (CSF). Such markers include components of the amyloid pathway $(A \beta 40, A \beta 42, A \beta 42 / A \beta 40$ ratio, and other truncated forms of $A \beta$ ) and of the tau protein pathway (tau and phosphotau). These CSF markers have been validated for the diagnosis of Alzheimer's disease, particularly early diagnosis, leading to harmonisation of procedures and markers between laboratories, in order to standardise CSF levels and to define decisional algorithms. Although these CSF biomarkers allow a positive diagnosis of disease state, there has been no demonstration of a link between disease progression and quantitative changes in these biomarkers, except in the early stage of the disease, where progression to Alzheimer's-type dementia is correlated with CSF levels of some biomarkers. ${ }^{[3]}$ This lack of association with progression is a limitation to their use as biomarkers of pharmacodynamic response and, a fortiori, as a surrogate marker for clinical response. The constraints of lumbar puncture have encouraged development of tests for the measurement of these same markers in plasma. Apart from markers specific to different pathways in Alzheimer's disease, other markers, which are indicative of nonspecific disease mechanisms (inflammation, oxidative stress, endothelial markers), could also potentially be useful as indicators of a physiopathological effect during the evaluation of "diseasemodifier" candidates which target non-specific disease pathways.

Imaging biomarkers are identified regularly due to technological advances in methodology, which have lead to a better understanding of the physiopathology of the disease. ${ }^{[4,5]}$ The two imaging approaches that have been most widely exploited are Magnetic Resonance Imaging (MRI) and nuclear imaging by 
Positron Emission Tomography (PET) or Single Photonic Computerized Tomography (SPECT). Two types of imaging biomarker can be distinguished. The first class corresponds to markers that are independent of physiopathology and that can be used to determine the topography of the neuronal lesions. These include MRI with volumetric analysis, PET-fluorodeoxyglucose (PET-FDG) and SPECT. Their utility has been indicated by the Alzheimer disease neuro imaging initiative (ADNI I) study, which showed that structural MRI and FDG-PET had greater statistical power than clinical and cognitive markers to detect change over time in a sample of subjects with Alzheimer's disease. ${ }^{[6]}$ The second class corresponds to specific (amyloid plaques, neurofibrillar degeneration) or non-specific (microglial activation) markers of the physiopathological process. Concerning metabolic imaging of the physiopathological process, radiotracers allow confirmation of amyloid lesions in the brain (PIB, AV45...), the presence of neurofibrillar degeneration (FDDNP) and of microglial activation (PK1195). These imaging markers are useful for diagnosis, since in cases of moderate amnesic-type cognitive decline, it is possible to make a diagnosis of "probable" Alzheimer's disease if hippocampal atrophy is visible on MRI or amyloid plaques detectable by PET. From a pharmacodynamic point of view, an association has been demonstrated between cognitive decline and progression of structural lesions on MRI, in particular global atrophy or hippocampal atrophy. On the other hand, the size of amyloid plaques does not appear to differ according to the evolutionary stage of the disease, leading to amyloid-PET being considered as a marker of disease state and not of progression. The role of PET in following the course of neurofibrillar degeneration and microglial activation is still under evaluation in basic research.

Although there are fewer data in the literature concerning neurophysiological biomarkers, they are still of considerable interest. They allow the identification of cerebral activity dysfunction in an objective and reproducible manner. Some anomalies of synchronisation on electroencephalography (EEG) may be associated with a risk of progression to Alzheimer's disease, recognised in cases of mild cognitive decline. It has been possible to correlate these electrophysiological anomalies with the severity of cognitive decline, although an association between disease progression and electrophysiological anomalies remains to be demonstrated.

\section{Phase II studies: different aspects of the study protocol}

Phase II studies have two principal objectives related to establishing proof of concept. The first is to demonstrate that the target chosen is relevant and the second to evaluate the doseresponse relationship in order to determine the dose to test in subsequent Phase III studies. Apart from defining these general objectives, the repeated failure of many drugs under development for Alzheimer's disease has led to a refinement of the design of Phase II trials with respect to the study population, assessment criteria, experimental design, and study duration. Two additional objectives should also be considered at this stage, firstly to demonstrate an effect on the physiopathology of the disease, as a function of the supposed mechanism of action of the candidate-drug, and secondly to generate safety data early on in the development programme, since certain target mechanisms may expose patients to an increased risk of serious adverse events, as the first trial with anti-beta immunotherapy demonstrated.

While the ultimate goal of a disease-modifying drug in Alzheimer's disease is to slow down the rate of cognitive decline, it is important that studies are better designed in order to distinguish candidate drugs that do not achieve a sufficient level of proof to justify continued development from those for which the demonstration of an effect is sufficiently robust to continue into Phase III development. In terms of target population, it is even more crucial during Phase II to evaluate candidate drugs in those patients who are most likely to derive a benefit from a disease-modifying treatment, namely those at an early stage of the disease. In contrast, in patients who already have dementia it is likely that the physiopathological process is already too far advanced to be modified pharmacologically. During Phase II, it is probably necessary to enrich the target population by taking into account genetic polymorphisms or the presence of vascular lesions, and even to stratify by such factors in order to identify potential responders and thus increase the chances of success during Phase III.

Concerning the endpoint criteria, there appears to be little value at this stage in focusing on clinical and cognitive criteria. In contrast, candidate drugs should be evaluated using methods that can demonstrate an effect on disease progression more accurately, and thus avoid the risk of demonstrating a purely symptomatic effect, which is always possible with clinical criteria. Although they can usefully distinguish between a symptomatic effect and a disease modifying effect, it is not appropriate to use "delayed start" type study designs in Phase II as they are poorly adapted to this stage of drug development. During Phase II, it seems most reasonable to use a classical protocol with parallel groups comparing the candidate treatment to placebo. Although short duration trials (3-6 months) will enable the demonstration of a significant effect for symptomatic treatments, longer treatment periods will be necessary to have a chance of demonstrating a change in the course of the disease. Given the rate of decline of cognitive function in the natural course of disease [approximately 3 points on the mini mental status (MMS) per year], it would be reasonable to consider a duration of one year for Phase II studied of potential disease modifying treatments. This raises the question of add-on 
Table I. Relative weight attributed to different biomarkers by Round Table $\mathrm{n}^{\circ} 3$ of Giens XXV (according to the conclusions of the Oxford Task Force, 2009). ${ }^{\text {[10] }}$

\begin{tabular}{|c|c|c|c|c|c|c|}
\hline & Type of biomarker & Diagnosis & $\begin{array}{c}\text { Supplementation of } \\
\text { study population }\end{array}$ & Stratification & $\begin{array}{c}\text { Evaluation } \\
\text { of effect }\end{array}$ & $\begin{array}{c}\text { Mechanism } \\
\text { of action }\end{array}$ \\
\hline \multirow{5}{*}{ Bio-CSF } & $\mathrm{A} \beta(40+42)$ & +++ & $?$ & + & + & +++ \\
\hline & Total tau & ++ & $?$ & - & + & ++ \\
\hline & Phos tau & ++ & ++ & + & + & +++ \\
\hline & IAT1 & +++ & $?$ & $?$ & + & +++ \\
\hline & BACE 1 & + & + & - & $?$ & +++ \\
\hline Bio-plasma & $\mathrm{A} \beta(40+42)$ & - & & - & + & ++ \\
\hline Bio-other & Inflammatory & - & - & - & - & + \\
\hline \multirow[t]{3}{*}{ Genetic } & Vascular & - & - & - & - & + \\
\hline & ApoE status & - & +++ & +++ & - & + \\
\hline & $\begin{array}{c}\text { Kits and sets } \\
\text { of genes }\end{array}$ & - & - & - & - & + \\
\hline \multirow[t]{5}{*}{ Imaging } & PET (amyloid) & +++ & +++ & ++ & ++ & +++ \\
\hline & FDG-PET & ++ & ++ & ++ & +++ & ++ \\
\hline & fMRI & - & - & - & + & - \\
\hline & SPECT & + & - & - & + & - \\
\hline & Structural MRI & ++ & + & ++ & $\begin{array}{c}+++ \\
(+ \text { tolerance }) \\
\end{array}$ & - \\
\hline Neurophysiology & EEG/evoked potentials & - & + & + & $?$ & - \\
\hline Cognitive & $\begin{array}{c}\text { Hippocampal amnesic } \\
\text { syndrome }\end{array}$ & +++ & +++ & + & ++ & - \\
\hline
\end{tabular}

A $\beta$ : amyloïd peptide; BACE 1: Beta site APP Cleaving Enzyme 1; EEG: electroencephalography; IATI: Innotext Amyloid Tau Index; MRI: Magnetic Resonance Imaging (fMRI: funcitonnal Magnetic Resonance Imaging); SPECT: Single Photonic Computerized Tomography.

therapy, because it is illusory to be able to maintain a patient with Alzheimer's disease in the prodromal stage or at the mild stage for one year without any treatment.

During Phase II studies, biomarkers should be used at all stages of the study design, namely for diagnosis, enrichment, stratification, evaluation, mechanism, and safety. Although clinical parameters are indispensable, it would be more beneficial to consider biological, genetic, imaging or neurophysiological biomarkers first. However, these biomarkers are not all relevant to all aspects of a Phase II study, requiring a critical analysis of their pertinence for a given study.

\section{Which biomarkers and for which parameters?}

Following a discussion by the expert panel, a relative weight was attributed, by consensus, to each type of biomarker for the different aspects of Phase II studies (table I). In the area of diagnosis, in addition to the existence of cognitive symptoms in the form of hippocampal amnesic syndrome, biomarkers of Alzheimer's disease-specific physiopathology appeared to be the most relevant, either biological markers such as $\mathrm{A} \beta 40,{ }^{[7]}$ tau and phosphotau in the CSF or imaging markers such as PET for the identification of amyloid plaques. At the second level, biomarkers of lesional topography (volumetric MRI, PET-FDG) could support the existence of a degenerative process in the early stages of the disease, thus helping in the positive diagnosis necessary for the inclusion in trials.

With respect to patient enrichment, one of the objectives is to be able to clarify the progressive phase of the disease using biomarkers that are modified during disease progression (CSFp-tau, CSF-t-tau, ${ }^{[7]}$ or A $\beta 42 / p$-tau 181 ratio, ${ }^{[8]}$ atrophy on MRI, alteration in metabolism on PET-FDG) or markers capable of indicating disease progression or response to treatment (PET-FDG which may be a marker of efficacy). Based on the possibility of enriching the profile of the population studied in a trial, stratification is probably necessary, depending on the criteria used to distinguish populations having different levels of response to the treatment under evaluation. By analogy with symptomatic treatment, stratification as a function of genetic polymorphism of apolipoprotein E could be proposed even if, in the future, it may be expected that other perhaps more interesting polymorphisms will be discovered. A second approach is to stratify according to the lesion burden, whether specific (amyloid-PET or CSF-tau, p-tau181 or increased tau(s)/A $\beta 42$ ratios) ${ }^{[3,8]}$ or non-specific (volumetric MRI, PET-FDG), starting with the hypothesis that patients with the least lesion progression could have a more significant modification of their disease course.

In terms of evaluation criteria, the expert panel did not consider that biological biomarkers were the most relevant at this 
Table II. Choice of biomarkers for Phase II clinical development.

\begin{tabular}{lcc}
\hline $\begin{array}{l}\text { Biomarkers for } \\
\text { diagnosis }\end{array}$ & $\begin{array}{c}\text { Biomarkers for } \\
\text { evaluation }\end{array}$ & $\begin{array}{c}\text { Biomarkers of } \\
\text { mechanism of action } \\
\text { (molecule dependant) }\end{array}$ \\
\hline $\begin{array}{l}\text { MRI+CSF } \\
\text { MRI+amyloid imaging }\end{array}$ & FDG & $\begin{array}{c}\text { CSF+plasma } \\
\text { vRI+FDG }\end{array}$ \\
\hline
\end{tabular}

CSF: cerebrospinal fluid; FDG: fluorodeoxyglucose; PET: Positron Emission Tomography; MRI: magnetic resonance imaging; vMRI: volumetric MRI.

stage, taking into account the impossibility of repeating lumbar puncture. The development and validation of kits for the detection of these markers in plasma would lead to a review of this position. PET-FDG and volumetric MRI, evidence of an evolving lesion without it being typed physiopathologically, are considered as the most relevant biomarkers as assessment criteria. In the absence of demonstrated evolution, amyloid PET was not retained as a biomarker at the second level. Clinical criteria were also considered as indicators of efficacy at the second level. With reference to meningo-encephalitis induced by the first attempts at amyloid immunotherapy, it is considered that morphological MRI could also be an indicator of safety. Participants also considered that new biomarkers to be used as assessment criteria need to be developed. From this viewpoint, functional MRI and neurophysiological markers could be studied in the future in the setting of the Pharmacog project, undertaken by the Innovative Medicine Initiative [European Union (EU) and European Federation of Pharmaceutical Industries and Association (EFPIA)]. With respect to demonstration of a mechanism of action, it was clear to participants that only biological or imaging biomarkers targeting a physiopathological cascade specific to Alzheimer's disease are relevant at the moment, with the reservation that measuring these markers is only useful for pharmacological approaches modulating these particular cascades.

\section{Choice of biomarkers for phase II and study design}

Once the most relevant biomarkers had been identified, participants focussed on defining the optimal design for Phase II studies based on selection criteria, assessment criteria and physiopathological effect criteria (table II). With respect to selection criteria, the aim should be to include patients in the prodromal stage as defined by the criteria of Dubois, ${ }^{[9]}$ which represents the population most likely to benefit from a disease-modifying treatment. If the treatment is specific to the physiopathological process of Alzheimer's disease which is currently considered the most likely, the amyloid cascade, inclusion criteria should include hippocampal amnesic syndrome associated with MRI demonstrating hippocampal atrophy and CSF analysis showing a decrease in concentration of $A \beta 42$ or $A \beta 42 / 40$ ratio and an increase in the concentration of phospho-tau. An alternative paraclinical picture could be MRI with hippocampal atrophy and the demonstration of amyloid plaques on PET; or MRI with hippocampal atrophy and hypometabolism on PET-FDG.

As regards assessment criteria, a consensus was reached that the main assessment criterion should be modification of the progression of non-specific lesions showing neuronal loss ("topographic markers"), due to data showing an association between cognitive decline and the progression of lesions visualised by morphological or metabolic imaging. PET-FDG appeared to be the most sensitive criterion to evaluate pharmacological activity. Volumetric MRI was considered as an alternative assessment criterion. The secondary assessment criterion should be clinical, more particularly the Alzheimer Disease Assessment Scale-Cognitive (ADAS-cog) which remains the reference measure. The choice of physiopathological criteria is open to choice by default, since existing validated biomarkers are restricted to markers of the amyloid cascade. Although these markers are relevant for pharmacological agents that modify this cascade, they are not relevant for other mechanisms of action. This new study concept does not contradict the guidelines of EMA, ${ }^{[1]}$ which are progressive and already recognise studies in Alzheimer's disease carried out with biomarkers that still require validation. The establishment of a clinical effect on cognitive and functional decline during Phase III studies is already recognised as an effect on disease progression. This, supported by an effect on a biological or imaging biomarker, could be used to demonstrate an effect on the pathological process for a disease-modifying treatment.

\section{Algorithm for the decision to progress to Phase III (Go/Nogo)}

In addition to the design of the study, participants also discussed the attitude to take the decision to continue development into Phase III, as a function of the results obtained for the primary and secondary endpoints chosen (table III). In an ideal situation, the candidate drug will be shown to have a beneficial effect on both the progression of lesions by imaging (PET-FDG, or atrophy on MRI) and on cognitive decline. In this case, the decision to continue to Phase III is evident. If the candidate drug has a beneficial effect on cognitive decline, without demonstrating an effect on imaging markers, the decision is easy to make because of the existence of a clinical benefit. The absence of an effect on an objective criterion such as cerebral lesions, leads to the risk that the benefit will turn out to be a symptomatic rather than a diseasemodifying effect. The existence of an effect on objective imaging 
Table III. Algorithm for the decision to enter Phase III development (Go/Nogo).

\begin{tabular}{lllll}
\hline FDG & + & - & + & - \\
ADASCog & - & + & + & - \\
Go/NoGo & Go & Go & Go & NoGo \\
\hline
\end{tabular}

ADAS-Cog: Alzheimer Disease Assessment Scale-Cognitive; FDG : Fluorodeoxyglucose.

criteria in the absence of a clinical effect may be more risky to consider as a priority for Phase III development. However, at this stage, clinical criteria may be less sensitive than imaging criteria, justifying taking the risk to continue into Phase III. Conversely, the participants considered that development should be stopped in the absence of a clinical effect or an effect demonstrated by imaging in order to avoid the loss of money and time studying an agent that has little chance of success.

\section{Conclusion}

In order to minimise failure during clinical development of molecules for the treatment of Alzheimer's disease, the objective is to limit the risk of failure during phase III by improving the quality of the Go/Nogo decision to proceed from Phase II into Phase III. This decision should no longer be based uniquely on clinical assessment criteria and in poorly selected patients presenting with disease that has already progressed. The consensus recommendations of the French experts was that Phase II studies should be carried out in patients presenting with the prodromal phase of Alzheimer's disease diagnosed by the presence of clinical criteria for hippocampal amnesic syndrome and biological and imaging biomarkers. The demonstration, at this stage of the disease, of a beneficial effect on the progression of non-specific lesional markers on imaging or on cognitive decline, or both, should support the decision to continue development, while recognising that the ideal situation is the demonstration of both a lesional and clinical effect. Conversely, the experts considered that there was no point in continuing the development into Phase III of a candidate- drug that has neither a clinical effect nor an effect on lesion progression. These views should help manufacturers in the design of Phase II studies and in their decision to continue development or not depending on the results. Participants considered that implementation of these recommendations will optimise the development of new molecules in Alzheimer's disease, will save time and money, and will avoid the risk of ending up, due to lack of success in clinical development, with discontinuation of industrial research in this field, as has happened in cerebrovascular disease.
Participants. Zeina Antoun (Laboratoire GlaxoSmithKline), Olivier Arnaud (Inserm Transfert, Paris), Olivier Blin (CICUPCET, Marseille), Antoine Coquerel (CHU Côte de Nacre, Caen), Catherine Deguines (Afssaps, Saint-Denis), Philippe Derambure (CHRU, Lille), Patrice Dosquet (Haute Autorité de Santé, Saint-Denis La Plaine), Jean-Pierre Duffet (DHOS-OPRC, Antony), Sylvia Goni (Laboratoire Lundbeck), Philippe Gustovic (Wyeth Pharmaceuticals), Marie Lang (Ministère de la Santé, Paris), Marie-Laure Laroche (CRPV, CHU Dupuytren, Limoges), Antoine Pariente (Inserm U 657, Bordeaux), Jean-Jacques Pere (Laboratoire Novartis), Odile Regnier (Cnamts, Paris), Philippe Truffinet (Laboratoire Sanofi Aventis).

\section{References}

1. CHMP 2008. Guideline on medicinal products for the treatment of Alzheimer's disease and other dementias. http: //www . emea. europa.eu/ pdfs/human/ewp/ $\mathrm{T} 1 \backslash 055395$ en.pdf

2. Lathia CD, Amakye D, Dai W, et al. State of the art. The value, qualification, and regulatory use of surrogate end points in drug development. Clinical Pharmacology \& Therapeutics 2009; 86 (1): 32-43

3. Snider BJ, Fagan AM, Roe C, et al. Cerebrospinal fluid biomarkers and rate of cognitive decline in very mild dementia of the Alzheimer type. Arch Neurol 2009; 66(5): 638-45

4. Jack CR, Bernstein MA, Borowski BJ, et al. Update on the magnetic resonance imaging core of the Alzheimer's disease neuroimaging initiative. Alzheimer's \& Dementia 2010; 6(3): 212-20

5. Jagust WJ, Bandy D, Chen K, et al. The Alzheimer's disease neuroimaging initiative positron emission tomography core. Alzheimer's \& Dementia 2010; 6(3): $221-9$

6. Walhovd KB, Fjell AM, Brewer J, et al. Combining MR imaging, positronemission tomography, and CSF biomarkers in the diagnosis and prognosis of Alzheimer disease. American Journal of Neuroradiology 2010; 31: 347 54

7. Van der Vlies AE, Verwey NA, Bouwman FH, et al. CSF biomarkers in relationship to cognitive profiles in Alzheimer Disease SYMBOL. Neurology 2009; 72(12): 1056-61

8. Fagan AM, Mintun MA, Shah AR, et al. Cerebrospinal fluid tau and ptau181 increase with cortical amyloid deposition in cognitively normal individuals: implications for future clinical trials of AD. EMBO Molecular Medicine 2009; 1(8-9): 371-80

9. Dubois B, Feldman HH, Jacova C, et al. Research criteria for the diagnosis of Alzheimer's disease: revising the NINCDS-ADRDA criteria. Lancet 2007; 6: 734-46

10. Vellas B, Andrieu S, Aisen P, et al. for the Oxford Task Force Group. Biomarkers for Alzheimer's disease therapeutic trials: The Oxford Task Force. Forthcoming

Correspondence and offprints: Claude Bidaut-Mazel, GlaxoSmithKline, Direction Médicale, 100 route de Versailles, 78163 Marly le Roi Cedex, France.

E-mail: claude.m.bidaut-mazel@gsk.com; claudebidaut-mazel@orange.fr 